\section{Inhibition des réponses immunitaires anti-tumorales par NF-KB}

\section{Vers un ciblage thérapeutique?}

Lara Revol-Bauz ${ }^{1}$, Yenkel Grinberg-Bleyer ${ }^{2}$
${ }^{1}$ Master de cancérologie, module

d'immunologie - virologie, université Claude Bernard Lyon 1, Lyon, France.

${ }^{2}$ Centre de recherche en cancérologie de Lyon, UMR Inserm 1052, CNRS 5286, centre Léon Bérard, université Claude Bernard Lyon 1, Lyon, France.

lara.revol----bauz@etu.univ-lyonl.fr Yenkel.grinberg-bleyer@inserm.fr

dans le mélanome chez la souris; de plus, ces composés potentialisent les effets de l'immunothérapie par anticorps anti-PD-1 (programmed cell death-1). Ces premières données mettent en évidence l'intérêt de la caractérisation précise de la fonction de chacune des sous-unités de NF- $K B$ en onco-immunologie. Ces travaux sur $\mathrm{c}$-Rel ont récemment été complétés par une analyse du rôle de NF-KBl.

\section{La sous-unité NF-KB1 promeut}

la progression du cancer colorectal chez la souris

Dans une étude publiée en 2020, Lu et al. se sont intéressés au rôle de la sous-unité NF-KBl sur la réponse lymphocytaire $T$ dans un modèle de cancer colorectal (CRC) induit par une inflammation chronique [6]. Avant toute stimulation cellulaire, $N F-\kappa B l$, protéine ubiquitaire, est retenue dans le cytoplasme dans sa forme entière, la protéine p105. Après stimulation, la forme clivée p50 de NF-kBl est transloquée dans le noyau où elle se lie au promoteur des gènes dont elle induit l'expression. Les auteurs ont observé tout d'abord par EMSA (electromotility shift assay) une forte activation de la voie NF- $\kappa B$, en particulier de p50, dans le côlon des souris atteintes de CRC. De même, l'analyse par immunohistochimie du microenvironnement tumoral de patients atteints de CRC a mis en évidence une activation de NF- $\mathrm{KBl}$ dans les cellules immunitaires et, dans une moindre mesure, dans les cellules tumorales. Ces résultats ont conduit à questionner le rôle de la sous-unité NF- $\kappa B l$ dans la tumorigenèse et la réponse immunitaire anti-tumorale. Pour étudier cette question, 


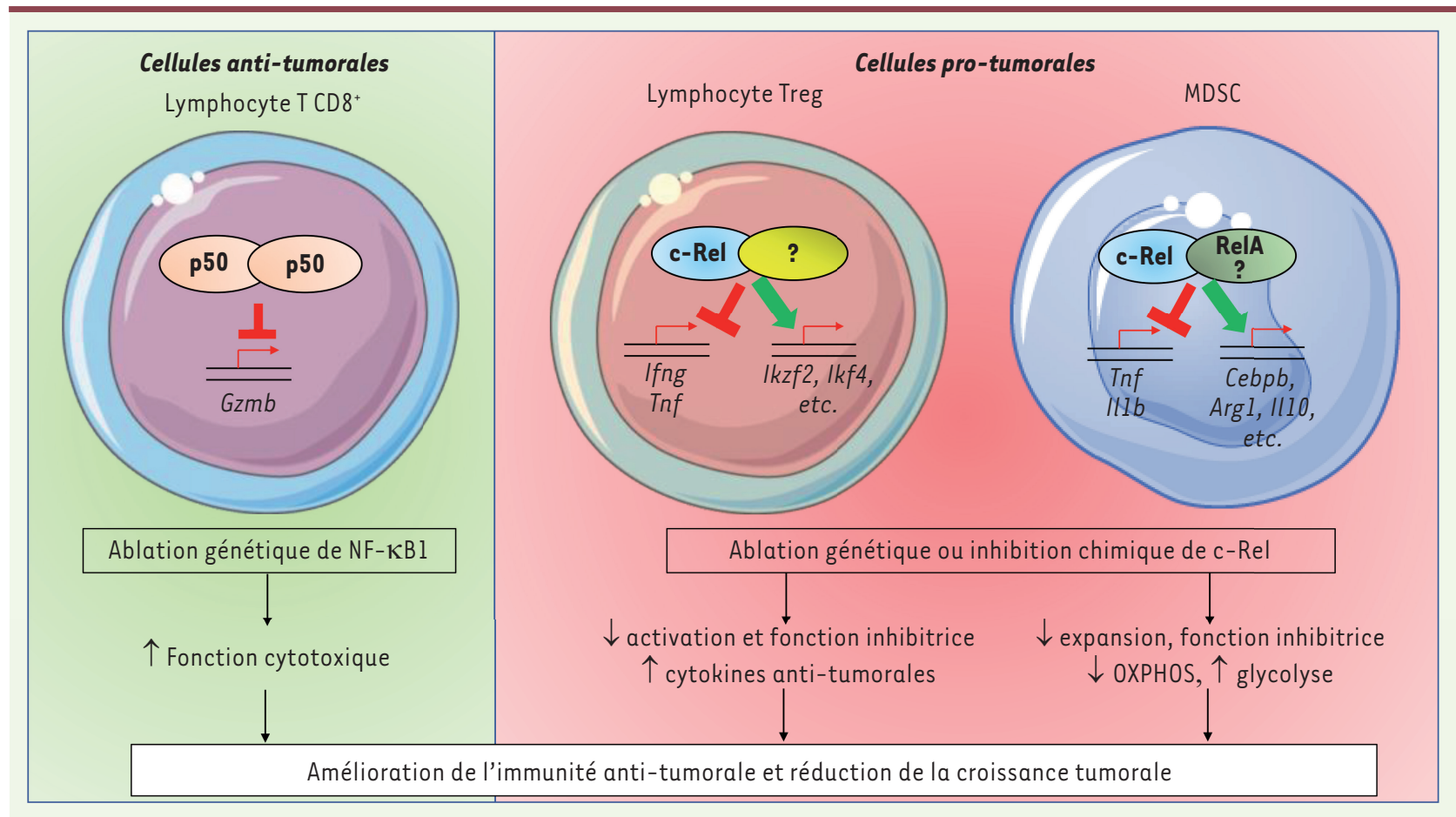

Figure 1. Rôles inhibiteurs de NF- KBI et c-Rel dans l'immunité anti-tumorale. Alors que NF- $\mathrm{KBl}$ inhibe la fonction cytotoxique des lymphocytes T CD8 ${ }^{+}$en réprimant l'expression du granzyme B, c-Rel favorise l'expansion et l'activité suppressive des lymphocytes T régulateurs (LTreg) et des MDSC (myeloid-derived suppressor cells) et inhibe leur expression de cytokines inflammatoires. En conséquence, l'ablation ou l'inhibition de c-Rel et de NF-KBl permet l'amélioration des réponses anti-tumorales chez la souris. OXPHOS : phosphorylation oxydative; Gzmb: granzyme B ; Ifng : interféron-gamma, Tnf : tumor necrosis factor-alpha; Ikzf2: Helios; Ikzf4: Eos; Illb: interleukine 1-beta ; Cebpb : CCAAT/enhancer-binding protein beta ; Argl : Arginase ; 1110 : interleukine-10.

Lu et al. ont alors analysé l'effet de la délétion du gène NFKBI (codant la sousunité $\mathrm{NF}-\kappa \mathrm{KB}$ ) sur la croissance tumorale. En utilisant un modèle de tumeur induite par plusieurs cycles de traitement par une combinaison de molécules qui provoque une inflammation du côlon et induit le développement de tumeurs (AOMDSS [azoxyméthane - dextran-sulfate de sodium]), ces chercheurs ont montré une réduction significative du nombre de nodules chez les souris Nfkbl-knock-out (KO) par rapport aux souris témoins. Des résultats similaires ont été observés dans un modèle d'adénocarcinome de côlon àù les cellules MC-38 sont injectées par voie sous-cutanée. Ces résultats suggèrent que $\mathrm{NF}-\kappa \mathrm{Bl}$ a un rôle pro-tumoral et favorise le développement du CRC. Dans ce modèle, la diminution de la croissance tumorale que l'on observe peut être liée à l'absence d'expression de $\mathrm{NF}-\mathrm{KBl}$ dans les cellules épithéliales de l'intestin ou dans les cel- lules du système immunitaire, ou dans les deux types de cellules. Pour répondre à cette question, les auteurs ont utilisé un modèle de chimères de moelle osseuse, pour restreindre l'invalidation de NFKBI au système hématopoḯtique. Ces expériences ont montré que les souris dont le gène $N F K B I$ a été invalidé spécifiquement dans les cellules hématopoiétiques, présentent significativement moins de nodules que les souris témoins. À l'inverse, l'ablation $\mathrm{du}$ gène dans les cellules tumorales, en utilisant la technique CRISPR/Cas9, semble accélérer leur croissance. Cela montre que l'expression de NF-KBl dans les cellules immunitaires a une fonction pro-tumorale.

\section{NF-KB1 inhibe l'expression du granzyme B par les lymphocytes T cytotoxiques}

Afin de comprendre les mécanismes moléculaires contrôlés par NF- $\mathrm{KBl}$ et responsables du contrôle de la crois- sance tumorale, les auteurs ont analysé le transcriptome et le phénotype immunitaire des tumeurs issues de souris témoins et de souris Nfkbl-KO traitées par AOM-DSS. Parmi les nombreux gènes dont l'expression est affectée par $N F-\kappa B l$, l'expression du gène codant le granzyme $B$ est significativement augmentée dans les tumeurs de souris $\mathrm{Nfkbl-KO}$, à la fois en termes de transcription en ARN et de traduction en protéine, alors que la proportion de LT CD $8^{+}$reste inchangée. Cela suggère que $N F-\kappa B l$ réprime l'expression $d u$ gène codant le granzyme $B$, une molécule importante pour l'activité cytotoxique des LT. Pour confirmer ces résultats, l'expression du granzyme B par les LT après restimulation in vitro, $a$ été évaluée par cytométrie en flux. À nouveau, les LT dont le gène NFKBI a été invalidé expriment un niveau plus important de granzyme B. En ce qui 
concerne les mécanismes moléculaires impliqués, les auteurs ont montré, par des expériences d'immuno-précipitation de la chromatine, que NF- $\kappa B l$ est capable de se fixer directement au promoteur du gène codant le granzyme $B$, suggérant une répression directe de la transcription du gène.

Les auteurs ont ensuite évalué la fonction de $N F-\kappa B l$ dans la réponse aux immunothérapies effectuées avec des anticorps anti-PD-1. Alors que l'anticorps anti-PD-1 induit une forte diminution du volume des tumeurs MC-38 dans des souris témoins, l'effet bénéfique de la délétion de NFKBI n'est pas accru par l'immunothérapie. Ces résultats suggèrent que l'ablation de $\mathrm{Nfkbl}$ et l'anticorps anti-PDl activent un programme cytotoxique similaire dans les LT. Enfin, à partir de leurs propres données, les auteurs établissent tout d'abord une signature de gènes contrôlés par NF- $\mathrm{BB} l$. La comparaison de cette signature spécifique avec la base de données TCGA, qui collecte les données transcriptomiques de patients atteints de cancers, montre une corrélation inverse entre l'expression du granzyme $B$ et la signature NF- $\kappa B l$ chez les patients atteints de CRC. Enfin, chez les patients atteints de mélanome et traités par un anticorps anti-PD-1 (nivolumab), on peut observer une baisse du score $\mathrm{NF}-\kappa \mathrm{Bl}$ et une augmentation de l'expression du granzyme $B$.

Ce travail apporte un éclairage nouveau sur le rôle spécifique de NF- $\kappa B l$ dans la réponse lymphocytaire $T$ anti-tumorale. Toutefois, certains mécanismes moléculaires et cellulaires restent à élucider:
- NF- $\kappa B l$, à la fois via sa forme native pl05 et sa forme active p50, semble agir comme un répresseur de l'activité des $\mathrm{LT} \mathrm{CD}^{+}$. À l'inverse, RelA et c-Rel sont des régulateurs «positifs» de nombreuses fonctions des LT, en particulier la prolifération et la production de cytokines comme I'IL-2 [7]. II semble donc indispensable d'élucider le rôle de ces deux sous-unités dans la fonction anti-tumorale des LT cytotoxiques, afin de conclure quant au rôle de NF- $\kappa B$ dans ce contexte.

- Dans l'étude de Lu et al., les fonctions intrinsèques de $N F-\kappa B$ dans les différentes sous-populations de LT, en particulier les LT CD8 ${ }^{+}$cytotoxiques, ne sont pas clairement élucidées. En effet, dans le modèle $\mathrm{N} f k b l-K O$, le gène est inactivé dans toutes les cellules de l'organisme. Les effets délétères de NF-KBl pourraient donc reposer sur d'autres populations immunitaires, notamment les LTreg ou les MDSC.

\section{Vers un ciblage des sous-unités de NF-KB pour une immunothérapie du cancer?}

Ces données suggèrent un rôle délétère de NF-KBl et de c-Rel dans la progression tumorale, via l'effet de ces deux facteurs de transcription dans différentes populations immunitaires (Figure 1). Cela fournit un nouveau rationnel pour le ciblage de ces protéines qui semble être prometteur pour développer une thérapie anti-cancéreuse. L'inhibition totale de NF-kB a déjà été envisagée à de multiples reprises dans le passé du fait du rôle délétère de la voie NF- $\kappa B$ dans la progression tumorale et le processus métastatique, mais le manque de spécificité de cette approche globale a représenté un frein majeur [8]. Afin de développer des traitements spécifiques et de s'assurer de leurs efficacité et de leur sûreté, il serait néanmoins important de disséquer la fonction des différentes sous-unités de $N F-\kappa B$, notamment celles impliquées dans la voie alternative, à la fois dans les cellules cytotoxiques, dans les cellules immunomodulatrices et dans les cellules tumorales elles-mêmes. $\diamond$

Inhibition of anti-tumor immunity by

NF-KB: Toward therapeutic targeting?

\section{LIENS D'INTÉRÊT}

Les auteurs déclarent n'avoir aucun lien d'intérêt concernant les données publiées dans cet article.

\section{RÉFÉRENCES}

1. Dubois M, Ardin C, Andre F, et al. L'immunothérapie, une révolution en oncologie - Revue de l'efficacité des inhibiteurs de points de contrôle immunitaire. Med Sci (Paris) 2019 ; $35: 937-45$.

2. Taniguchi $K$, Karin M. NF- $\kappa B$, inflammation, immunity and cancer: coming of age. Nat Rev Immunol 2018 $18: 309-24$.

3. Bruchard M, Ghiringhelli F. Microenvironnement tumoral : cellules régulatrices et cytokines immunosuppressives. Med Sci (Paris) 2014 ; $30: 429-35$.

4. Grinberg-Bleyer $\mathrm{Y}, \mathrm{Oh} \mathrm{H}$, Desrichard A, et al. NF- $\mathrm{KB}$ $c$-Rel is crucial for the regulatory $T$ cell immune checkpoint in cancer. Cell 2017 ; 170 : 1096-108.el3.

5. Li T, Li X, Zamani A, et al. c-Rel is a myeloid checkpoint for cancer immunotherapy. Nat Cancer 2020; $1:$ 507-17.

6. Lu C, Klement JD, Smith AD, et al. p50 suppresses cytotoxic T lymphocyte effector function to regulate tumor immune escape and response to immunotherapy. J Immunother Cancer 2020 ; 8 : e001365.

7. Lalle G, Twardowski J, Grinberg-Bleyer Y. NF-kappaB in cancer immunity: friend or foe? Cells 2021; $10: 355$.

8. Baud V, Karin M. Is NF-kappaB a good target for cancer therapy? Hopes and pitfalls. Nat Rev Drug Discov 2009 ; 8 : 33-40.
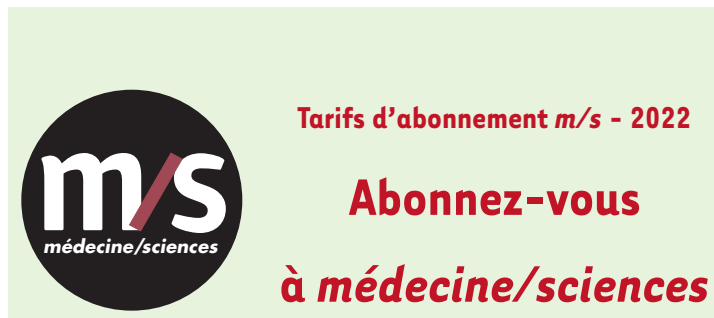

$>$ Grâce à $m / s$, vivez en direct les progrès des sciences biologiques et médicales

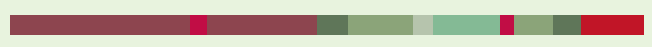

à médecine/sciences

Abonnez-vous sur www.medecinesciences.org

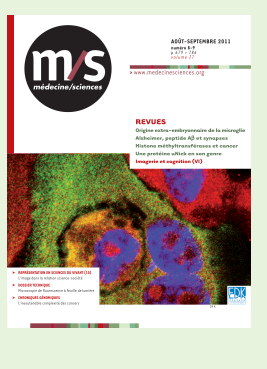

